\title{
The human gut microbiome: the ghost in the machine
}
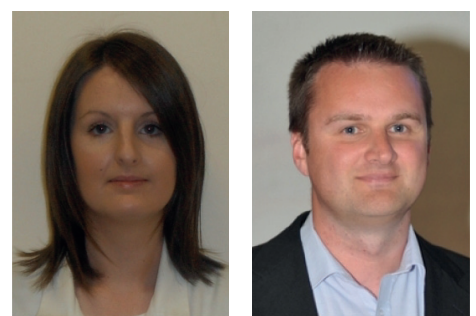

“...this gut-associated microbial conglomerate ... represents a vital virtual organ, integrally linked to our physical and mental health and wellbeing."

Audrey Feeney' \& Roy D Sleator*1

'Department of Biological Sciences, Cork Institute of Technology, Rossa Avenue, Bishopstown, Cork, Ireland *Author for correspondence: Tel. : +353 214335405 w Fax: +353 214326885 wroy.sleator@cit.ie

Since the publication of the first complete genome of a free living organism, Haemophilus influenza, in 1995, the genomics era has experienced more than its share of triumphs and tribulations, culminating in the publication of our own draft human genome sequence in 2001. However, even this achievement is diminished when one considers that, despite the existence of an estimated 7 billion humans on Earth, we constitute less than $0.1 \%$ of the planet's total biomass. Microorganisms, on the other hand, make up approximately $50 \%$ of the Earth's biomass. Furthermore, our own resident microbial communities (more usually referred to as the microbiota) outnumber our human cells by a factor of ten and harbor at least 100-times more genetic information than the human genome [1]. Distilling this further, the human distal gut has been identified as one of the most densely populated ecosystems on Earth, containing more bacterial cells than all of our other microbial communities combined [2]. It should come as little surprise then that this gut-associated microbial conglomerate, with a metabolic potential far in excess of that of the gut itself, represents a vital virtual organ, integrally linked to our physical and mental health and wellbeing [3].

“...the Human Microbiome Project was initiated by the NIH as a worldwide initiative to gain a greater understanding of the genetic information contained within the gut microflora and what effect, if any, this information might have on human health and disease."

While the early pioneers of microbial gut ecology were largely restricted to culture-based approaches in order to study microbial gut communities, the tools and techniques developed as part of the Human Genome Project proved to be just as powerful in the postgenomics era. Indeed, recent significant advances in the development of these next-generation sequencing technologies are themselves worthy of a separate discussion, but this sadly falls outside the scope of the current editorial - we refer the reader to the review by Quail et al. [4].

So what constitutes postgenomics? For us, it is the shifting focus away from a single genome or organism towards entire communities in a specific environmental niche or symbiotic partnership (e.g., the human superorganism) [5]. Metagenomics, the functional and sequence-based analysis of the collective microbial genomes (microbiome) in a particular environment, represents such a shift and, as such, represents the most logical extension of the Human Genome Project [1]. Metagenomics now represents a core component of the many 'omics' technologies that constitute the newly established and rapidly expanding field of systems biology - an approach that favors a holistic, rather than reductionist, approach to studying complex ecosystems, such as the human distal gut. The first comprehensive human gut metagenomic study was carried out in 2005 [6]. Mucosal biopsy and fecal samples from three healthy adults were collected and 13,333 16S rRNA sequences were generated, resulting in the largest amount of sequencing data generated in a single study of any environment at that time. Of the 395 phylotypes identified, $48 \%$ were Bacteriodetes and $51 \%$ were Firmicutes, with the remaining microflora containing members of the Proteobacteria, Verrucomicrobia, Fusobacteria, Cyanobacteria, Spirochetes and VadinBE9V. Two years later, and with a whetted appetite, the Human Microbiome Project (HMP) was initiated by the NIH as a worldwide initiative to gain a greater understanding of the genetic information contained within the gut microflora and what effect, if any, this information might

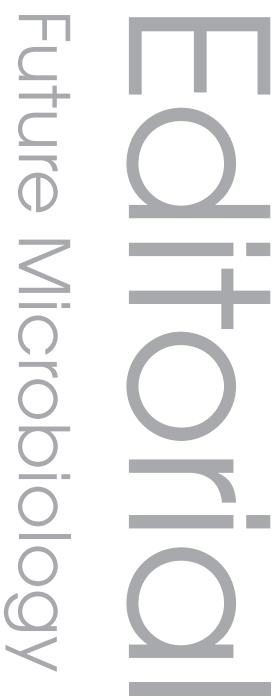

\section{Keywords}

- genomics $=$ gut

- metagenomics

- microbiome n microbiota

- pharmabiotics = probiotic

Future Medicine part of 
have on human health and disease [7]. In 2010, Nelson $e t$ al. published the results of an initial reference genome study of 178 genomes from the human gut microflora; this resulted in 30,867 polypeptides, of which 29,987 ( 97\%) were considered novel, providing a tantalizing glimpse into the potential treasure trove of novel genetic loci that exists within the gut microbiome [8]. The HMP consortium recently generated the largest and as yet most comprehensive reference set of human microbiome data consisting of 240 healthy individuals, spanning five major habitats and generating over 3.5 terabases of metagenomic sequence [9] (enough to fill 3.5 million textbooks of nucleotide sequence!).

Other more targeted studies focusing on specific populations of differing ages, genders, ethnicities and levels of obesity and fitness, among others, will likely reveal even more information about the microbiome and its potential impact on our overall health and wellbeing. A number of recent studies, for example, have focused specifically on changes in gut microbial communities that are linked to age [10]. Although physiological changes in the gastrointestinal (GI) tract coupled with decaying dentition can have a significant impact on the diversity of the gut microbiota of the aging population, diet has been identified as a major contributory factor, leading to a less diverse microbial community in the GI tract of the elderly [11]. Claesson et al. recently studied the microbiota of 178 elderly individuals, demonstrating that elderly people in long-stay care facilities showed significantly less diversity in their gut microbiota, as a result of a less varied diet, when compared with those living in the community, where a more varied diet was available [11]. The study demonstrated that loss of 'communityassociated microbiota' corresponded with markers of inflammation and increased frailty in these subjects. Interestingly, this and previous studies have demonstrated a greater interindividual variation in the gut microbiota of elderly individuals when compared with younger adult subjects $[10,11]$. When one considers that the US Consensus Bureau has predicted a worldwide population of 1.3 billion elderly individuals ( $\geq 65$ years of age) by 2040 , resulting in more elderly people than children less than 5 years of age in the world, it seems inevitable that already struggling health systems are likely to be stretched beyond capacity in the not-too-distant future. Thus, a greater understanding of the human gut microbiota, and tailoring diets accordingly, may help to improve health and reduce pressure on already strained health systems.
Imbalances in the composition of the human gut microbiome have been linked with chronic conditions such as Crohn's disease, obesity, cancer and inflammatory disorders including irritable bowel syndrome (IBS), one of the most commonly diagnosed GI conditions, affecting a reported $10-15 \%$ of individuals living in the USA. Indeed, the microbial communities that inhabit the GI tract have previously been implicated as potential triggers of IBS [12]. A recent study by Devkota et al. suggests that western diets, which are high in animal-derived saturated fats, have a significant effect on the gut microbiota in mice [13]; particularly, it promotes the growth of Bilophila wadsworthia, an organism that thrives on taurocholic acid (a sulfurcontaining molecule made of bile acid and taurine), which is more readily available during the ingestion of milk fats. The growth of B. wadsworthia induces a proinflammatory Th1 immune response, resulting in an increased incidence of colitis in genetically susceptible mice (with wild-type animals remaining unaffected). This study highlights the importance of including information on our individual genomes, coupled with that of our microbiome, in order to make informed decisions with regards to diet and nutrition, with a view to preventing diseases such as IBS. Although in its early stages, the potential for biomarker-based identification of at-risk individuals is obvious and will likely play a significant role in tailoring our dietary recommendations in the future.

"... a greater understanding of the human gut microbiota, and tailoring diets accordingly, may help to improve health and reduce pressure on already strained health systems."

While functional and sequence-based analysis of the human microbiome (and associated host interactions) has the potential to prevent diseases in genetically susceptible individuals through modifications of dietary recommendations [14], other more direct dietary interventions are also possible. In other words, rather than tailoring our food (prebiotic) intake to indirectly regulate the levels of beneficial bacteria in our gut, thereby improving health, why not ingest the bacteria (such as the probiotic Bifidobacterium breve [15]) directly? The literature is replete with examples of the potential health benefits of probiotic bacteria, such as alleviating the symptoms of chronic GI 
disorders and associated sequelae, combating infection and modulating severe autoimmune conditions [16]. In recent years, probiotics have begun to emerge from the shadows of 'snake oil medicines' and are finally beginning to represent a real and viable alternative to traditional drug-based therapies [17]. Furthermore, recent developments in synthetic and systems biology, based on the rapidly advancing 'omics' technologies, coupled with powerful computeraided bioinformatics and genetic engineering tools, will ultimately lead to the development of ever more sophisticated algorithms for whole-cell modeling [18], synthetic cell synthesis (of which JCVI-syn1.0 [19] is the progenitor) and eventually to a new class of pharmabiotics assembled from modular components of various origins (both human and microbe) and tailored to fulfill all the requirements of an ideal therapeutic/prophylactic agent [20].

In the immortal words of Robert Browning:

"Truth lies within ourselves..."

\section{Financial \& competing interests disclosure}

A Feeney is the recipient of an IRCSET EMBARK

Postgraduate Scholarship RS/2010/2300. RD Sleator

is an ECSMID research fellow. The authors have no other relevant affiliations or financial involvement with any organization or entity with a financial interest in or financial conflict with the subject matter or materials discussed in the manuscript apart from those disclosed.

No writing assistance was utilized in the production of this manuscript.

\section{References}

1. Sleator RD, Shortall C, Hill C. Metagenomics. Lett. Appl. Microbiol. 47(5), 361-366 (2008).

2. Culligan EP, Sleator RD, Marchesi JR, Hill C. Functional metagenomics reveals novel salt tolerance loci from the human gut microbiome. ISME J. 6(10), 1916-1925 (2012).

3. Culligan EP, Marchesi JR, Hill C, Sleator RD. Mining the human gut microbiome for novel stress resistance genes. Gut Microbes 3(4), 394-397 (2012).

4. Quail MA, Smith M, Coupland P et al. A tale of three next generation sequencing platforms: comparison of Ion Torrent, Pacific Biosciences and Illumina MiSeq sequencers. BMC Genomics 13, 341 (2012).

5. Sleator RD. The human superorganism of microbes and men. Med. Hypotheses 74(2), 214-215 (2010).

6. Eckburg PB, Bik EM, Bernstein CN et al. Diversity of the human intestinal microbial flora. Science 308(5728), 1635-1638 (2005).

7. Turnbaugh PJ, Ley RE, Hamady M, Fraser-Liggett CM, Knight R, Gordon JI. The Human Microbiome Project. Nature 449(7164), 804-810 (2007).
8. Nelson KE, Weinstock GM, Highlander SK et al. A catalog of reference genomes from the human microbiome. Science 328(5981), 994-999 (2010).

9. Human Microbiome Project Consortium. A framework for human microbiome research. Nature 486(7402), 215-221 (2012).

10. Claesson MJ, Cusack S, O'Sullivan et al. Composition, variability, and temporal stability of the intestinal microbiota of the elderly. Proc. Natl Acad. Sci. USA 108(Suppl. 1), 4586-4591 (2011).

11. Claesson MJ, Jeffery IB, Conde S et al. Gut microbiota composition correlates with diet and health in the elderly. Nature 488(7410), 178-184 (2012).

12. Shanahan F. The colonic microbiota and colonic disease. Curr. Gastroenterol. Rep. 14(5), 446-452 (2012).

13. Devkota S, Wang Y, Musch MW et al. Dietary-fat-induced taurocholic acid promotes pathobiont expansion and colitis in $1110^{-1-}$ mice. Nature 487(7405), 104-108 (2012).

14. Mcknite AM, Perez-Munoz ME, Lu L et al. Murine gut microbiota is defined by host genetics and modulates variation of metabolic traits. PLoS One 7(6), e39191 (2012).
15. Sheehan VM, Sleator RD, Hill C, Fitzgerald GF. Improving gastric transit, gastrointestinal persistence and therapeutic efficacy of the probiotic strain Bifidobacterium breve UCC2003. Microbiology 153(Pt 10), 3563-3571 (2007).

16. Sleator RD. Probiotics - a viable therapeutic alternative for enteric infections especially in the developing world. Discov. Med. 10(51), 119-124 (2010).

17. Culligan EP, Hill C, Sleator RD. Probiotics and gastrointestinal disease: successes, problems and future prospects. Gut Pathog. 1(1), 19 (2009).

18. Karr JR, Sanghvi JC, Macklin DN et al. A whole-cell computational model predicts phenotype from genotype. Cell 150(2), 389-401 (2012).

19. Sleator RD. The story of Mycoplasma mycoides JCVI-syn1.0: the forty million dollar microbe. Bioeng. Bugs 1(4), 229-230 (2010).

20. Sleator RD, Hill C. Rational design of improved pharmabiotics. J. Biomed. Biotechnol. 2009, 275287 (2009). 